\title{
Kahler's Disease Revealed by a Fracture of the Humeral Diaphysis
}

\author{
Dehayni B ${ }^{1 *}$, Amarir $\mathbf{M}^{2}$, Zaddoug 01, El Nouali², Benchakroune $\mathbf{M}^{1}$, \\ Zine $\mathbf{A}^{1}$, Tanane $\mathbf{M}^{1}$ and Jaafar $\mathbf{A}^{1}$ \\ ${ }^{1}$ Traumatology and Orthopaedics Department, Mohamed V Military Hospital, \\ Morocco
}

2Medical Imaging Department Mohamed V Military Hospital, Morocco

Research Article

Volume 3 Issue 3

Received Date: April 05, 2019

Published Date: April 26, 2019

DOI: $10.23880 /$ jobd-16000181

*Corresponding author: Badreddine Dehayni, Traumatology Service - Orthopaedics I, Mohamed V Military Hospital, Morocco, Tel: +212661408957; Email: docteurdehayni@gmail.com

\section{Abstract}

Kahler's disease or multiple myeloma is a malignant hematological pathology, characterized by the malignant proliferation of plasma cells. Reporting a case of a humeral shaft fracture in a non-traumatic context that revealed Kahler's disease during paraclinical investigations. Management involves histological confirmation of the pathology, osteosynthesis of the fracture by static centromedullary nailing, with medical treatment made of biphosphonate combined with a chemotherapy protocol targeting the disease. The management of a pathological fracture is a diagnostic emergency, but never an operating emergency. A diagnosis of certainty must be made before any intervention.

Keywords: Kahler Disease; Pathological Fracture; Humeral Diaphysis; Centromedullary Nail; Chemotherapy

\section{Introduction}

Kahler's disease or multiple myeloma is a malignant hematological pathology, characterized by the malignant proliferation of plasma cells. The most common symptomatology is osteolytic bone damage, severe bone pain, hypercalcemia, kidney damage and anemia. The occurrence of pathological fractures in the humeral diaphysis as the first clinical sign of multiple myeloma is rare.

We reported a case of a fractured humeral shaft that revealed Kahler's disease during paraclinical investigations.

\section{Materials and Methods}

This is a 73-year-old male with a history of high blood pressure under treatment, who had consulted the emergency department for acute left arm pain with total functional impotence of the left upper limb without any notion of trauma. The clinical examination on admission found a conscious and hemodynamically and respiratory stable patient with a swollen, deformed and painful left arm on palpation, the vascular and nervous examination of the left upper limb was normal. An X-ray of the arm taking the shoulder and elbow from the front and profile was performed, which showed the presence of a fracture of the upper third of the left humeral diaphysis on an osteolysis image with fuzzy limits without invasion of the soft tissues (Figure 1). 


\section{Journal of Orthopedics \& Bone Disorders}

An X-ray of the skull showed incomplete "cookie cutter" lesions (Figure 2). Without obvious lesions on a pelvic X-ray, a complement by an MRI of the left arm confirmed a fracture on a tumour process of the humeral diaphysis (Figure 3). The non-traumatic mechanism of the fracture as well as radiographic images led us to diagnose an unstable diaphyseal fracture on pathological bone. Serum protein electrophoresis showed the presence of a monoclonal peak migrating into the beta-2 globulin zone with a monoclonal band of IgA lambda isotypy (Figure 4). Kahler disease was confirmed on surgical biopsy. The patient was treated surgically with intramedullary osteosynthesis by locking nail with immobilization by elbow sling to the body for 03 weeks followed by functional rehabilitation (Figure 5). Bisphosphonate treatment has been initiated.
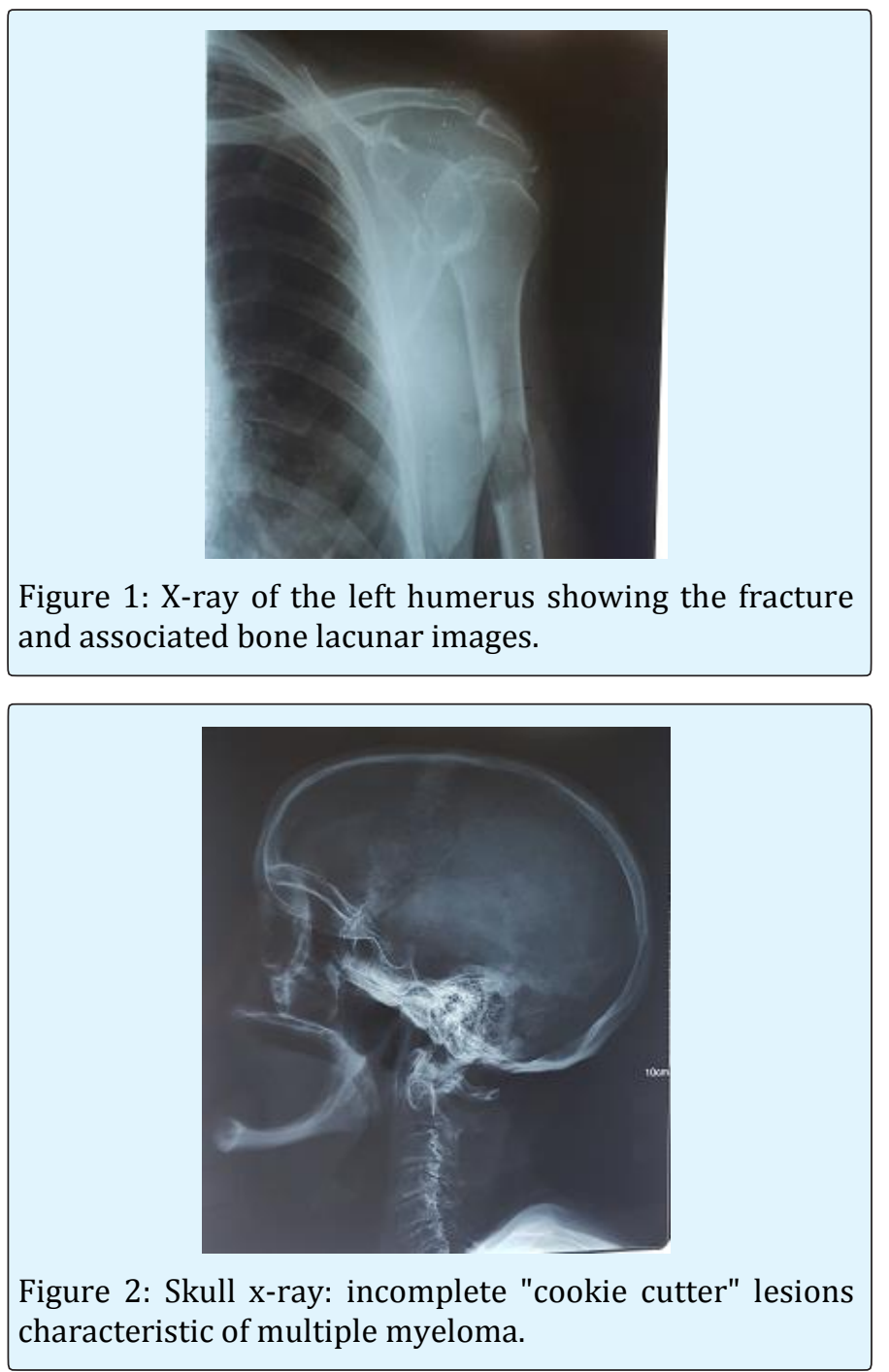

Dehayni B, et al. Kahler's Disease Revealed by a Fracture of the Humeral Diaphysis. J Ortho Bone Disord 2019, 3(3): 000181.
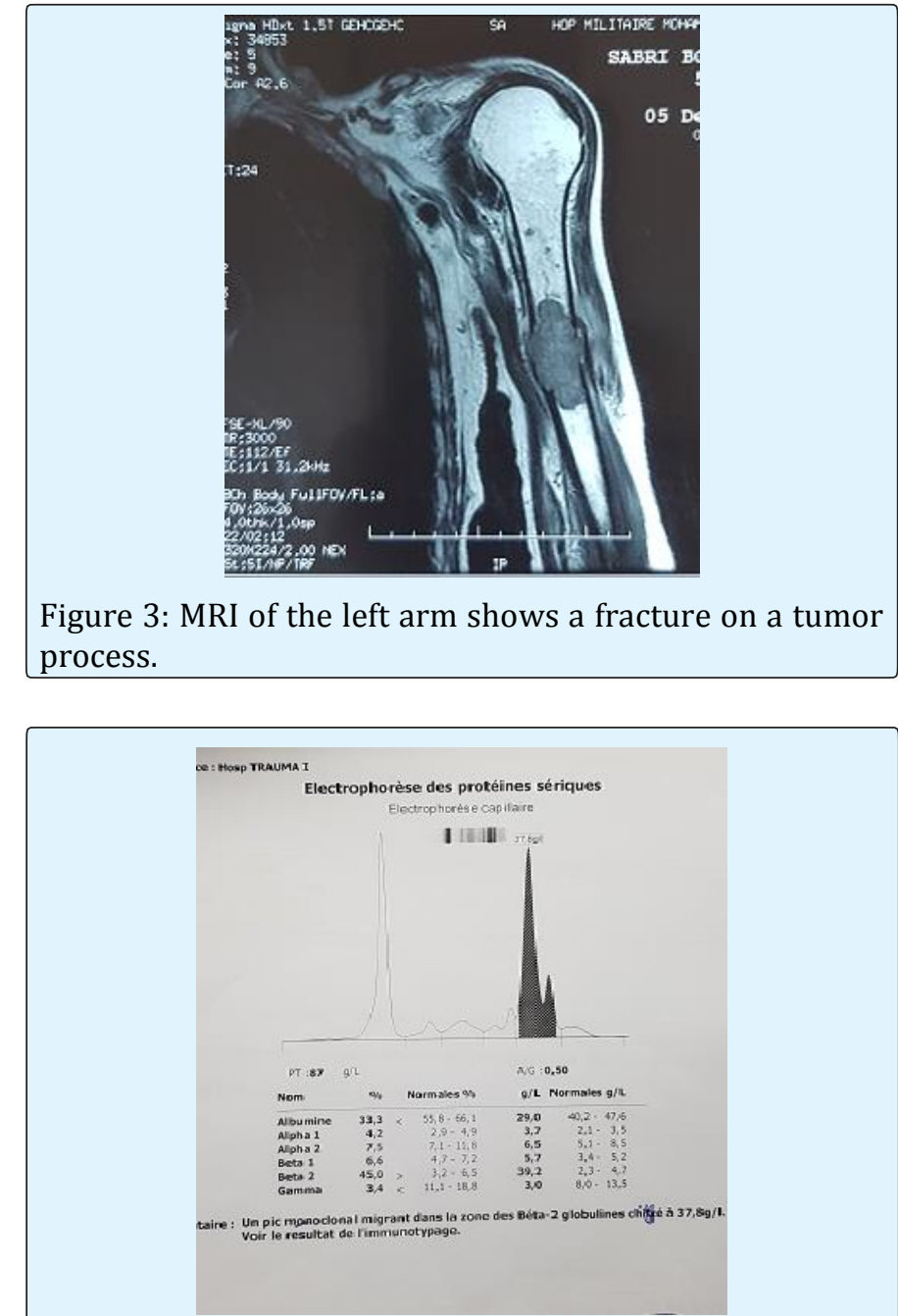

Figure 4: Serum protein electrophoresis: narrow peak migrating in the gamma globulin zone.

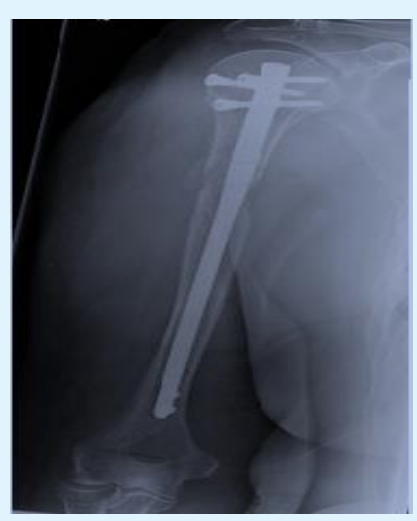

Figure 5: X-ray of the left humerus from the front showing good bone stabilization with a static nail. 


\section{Journal of Orthopedics \& Bone Disorders}

\section{Results}

The patient was referred to the oncologist for an induction chemotherapy protocol (bortezomib, thalidomide and dexamethasone). After a 6-month retreat, the patient was seen again in consultation in good general condition, the fracture is consolidated with a good functional recovery of his left upper limb. The patient's autonomy was decisive for the possibility of performing an auto graft of the bone marrow after induction chemotherapy and therefore for the control of his malignant hematopathy.

\section{Discussion}

Myeloma is a disease characterized by the development in the skeleton of multiple osteolytic plasma cell tumours (plasmocytomas) secreting a single monoclonal immunoglobulin in $80 \%$ of cases, either type G (twothirds of cases) or type A (one-third of cases). It is considered to be the most common malignant hematopathy after lymphoma, accounting for about $10 \%$ of blood cancers [1]. Bone involvement secondary to bone marrow infiltration is the most common manifestation and results in osteolytic lesions, bone pain and pathological fractures.

Fractures caused by myeloma can be managed in the same way as those caused by carcinoma, and their biological behaviour and mechanical implications are similar to metastatic fractures [2].

Bone metastases affecting the proximal humerus can be treated by arthroplasty resection or curettage of the pathological lesion, polymethyl methacrylate cement (PMMA) and plate osteosynthesis [3]. Prosthetic replacement provides excellent analgesic control, but often sacrifices part of the shoulder function [4]. This option will be indicated for patients with a sufficiently long vital prognosis, especially in the case of single metastasis. Stabilization of the pathologic fracture by PMMA-reinforced locked plate provides adequate local tumor control, good mechanical stabilization and satisfactory preservation of function $[5,6]$. Centromedullary nailing makes it possible to treat the majority of diaphyseal metastases, possibly reinforced by PMMA. It offers a good analgesic effect, allows rapid mobilization and with fewer complications [7-12]. This is the case for our patient but without PMMA. Bisphosphonate supplement therapy is essential to prevent and treat bone damage during kahler disease, whether oral clodronic acid or intravenous zoledronic or pamidronic acid [8,9]. The American Society for Clinical Oncology set the terms of this treatment in 2007, with a preference for intravenous bisphosphonates and minimum treatment duration of 2 years to be reconsidered based on the hematological response to auto-transplantation [10].

The anatomical and functional results in our patient after a six-month retreat, obtained by a stable (locked centromedullary nail), effective and durable osteosynthesis, resulted in a return to autonomy [11]. Katagiri prognostic survival score for patients with bone metastases is the most widely used in the literature. In our context a survival rate is estimated at $98 \%$ at 06 months and $75 \%$ at 24 months $[13,14]$.

\section{Conclusion}

Kahler's disease is a plasmocytic, malignant, clonal proliferation. Tumor plasma cells stimulate bone resorption responsible for bone gaps with the risk of pathological fracture. The management of a pathological fracture is a diagnostic emergency, but never an operating emergency. A diagnosis of certainty must be made before any intervention. The management of these fractures requires fixation by stable osteosynthesis, which is effective with fewer complications and allows the patient to regain his autonomy combined with an appropriate chemotherapy protocol

\section{Conflict of Interest}

The authors have no conflict of interest to declare

\section{References}

1. Kyle RA, Gertz MA, Witzig TE, Lust JA, Lacy MQ, et al. (2003) Review of 1027 patients with newly diagnosed multiple myeloma. Mayo Clin Proc 78(1): 21-33.

2. Jacofsky DJ, Haidukewych GJ (2004) Management of pathologic fractures of the proximal femur: state of the art. J Orthop Trauma 18(7): 459-469.

3. Du ZY, Zang J, Tang XD, Guo W, Chinese Orthopaedic Association Bone Oncology Group (2010) Experts' agreement on therapy for bone metastases. Orthop Surg 2(4): 241-253.

4. Ashford R, Benjamin L, Pendlebury S, Paul D Stalley (2012) The modern surgical and non-surgical 


\section{Journal of Orthopedics \& Bone Disorders}

management of appendicular skeletal metastases. Orthopaedics and Trauma 26(3): 184-199.

5. Siegel HJ, Lopez-Ben R, Mann JP, Ponce BA (2010) Pathological fractures of the proximal humerus treated with a proximal humeral locking plate and bone cement. J Bone Joint Surg Br 92(5): 707-712.

6. Weiss KR, Bhumbra R, Biau DJ, Griffin AM, Deheshi B, et al. (2011) Fixation of pathological humeral fractures by the cemented plate technique. J Bone Joint Surg Br 93(8): 1093-1097.

7. Redmond BJ, Biermann JS, Blasier RB (1996) Interlocking intramedullary nailing of pathological fractures of the shaft of the humerus. J Bone Joint Surg Am 78(6): 891-896.

8. Lahtinen R, Laakso M, Palva I, Virkkunen P, Elomaa I (1992) Randomized, placebo controlled multicentre trial of clodronate in multiple myeloma. Lancet 340(8827): 1049-1052.

9. Rosen LS, Gordon D, Kaminski M, Howell A, Belch A, et al. (2001) Zoledronic acid versus pamidronate in the treatment of skeletal metastases in patients with breast cancer or osteolytic lesions of multiple myeloma: a phase III, double blinded, comparative trial. Cancer J 7(5): 377-387.

10. Kyle RA, Yee GC, Somerfield MR, Flynn PJ, Halabi S, et al. (2007) American Society of Clinical Oncology 2007 clinical practice guideline update on the role of bisphosphonates in multiple myeloma. J Clin Oncol 25(17): 2464-2472.

11. Miller BJ, Soni EE, Gibbs CP, Scarborough MT (2011) Intramedullary nails for long bone metastases: why do they fail? Orthopedics 34(4).

12. Ruggieri P, Mavrogenis AF, Casadei R, Errani C, Angelini A, et al. (2010) Protocol of surgical treatment of long bone pathological fractures. Injury 41(11): 1161-1177.

13. Sonmez M, Akagun T, Topbas M, Cobanoglu U, Sonmez B, et al. (2008) Effect of pathologic fractures on survival in multiple myeloma patients: a case control study. J Exp Clin Cancer Res 27: 11.

14. Katagiri H, Takahashi M, Wakai K, Sugiura H, Kataoka, et al. (2005) Prognostic factors and a scoring system for patients with skeletal metastasis. J Bone Joint Surg Br 87(5): 698-703. 